\title{
ON THE SETWISE CONVERGENCE OF SEQUENCES OF MEASURES
}

\author{
JEAN B. LASSERRE \\ $L A A S-C N R S, 7$ Av. du Colonel Roche \\ 31077 Toulouse Cedex, France \\ lasserre@laas.fr
}

(Received April, 1996; Revised July, 1996)

\begin{abstract}
We consider a sequence $\left\{\mu_{n}\right\}$ of (nonnegative) measures on a general measurable space $(X, \mathfrak{B})$. We establish sufficient conditions for setwise convergence and convergence in total variation.
\end{abstract}

Key words: Measures, Convergence of Sequences of Measures.

AMS subject classifications: 28A33, 28C15.

\section{Introduction}

Consider a sequence $\left\{\mu_{n}\right\}$ of (nonnegative) measures on a measurable space $(X, \mathfrak{B})$ where $X$ is some topological space. Setwise convergence of measures, as opposed to weak* $^{*}$ or weak convergence, is a highly desirable and strong property. If proved, some important properties can be derived (for instance, the Vitali-Hahn-Saks Theorem) and thus sufficient conditions ensuring this type of convergence are of interest. However, as noted in [2], in contrast to weak or weak ${ }^{*}$ convergence (for instance, in metric spaces), it is in general difficult to exhibit such a property, except if e.g., $\mu_{n}$ is an increasing or decreasing sequence (e.g. [2], [4]).

The present paper provides two simple sufficient conditions. Thus, for instance, in a locally compact Hausdorff space, an order-bounded sequence of probability measures that is vaguely or weakly convergent is in fact setwise convergent.

We also establish a sufficient condition for the convergence in total variation norm that is even a stronger property.

\section{Notations and Definitions}

Let $(X, \mathfrak{B})$ be a measurable space and let $\mathcal{M} b(X)$ denote the Banach space of all bounded measurable real-valued functions on $X$ equipped with the sup-norm. Let $S$ be the positive (convex) cone in $\mathcal{M} b(X)$.

Let $\mathcal{M b} b(X)^{\prime}$ be the (Banach) topological dual of $\mathcal{M} b(X)$ with the duality bracket $\langle\cdot, \cdot\rangle$ between $\mathcal{H} b(X)$ and $\mathcal{M} b(X)^{\prime} . \quad \mathcal{L} b(X)^{\prime}$ is equipped with the dual norm $|\varphi|:=\sup _{\|f\|=1}|\langle\varphi, f\rangle|$. Let $S^{\prime} \in \mathcal{M} b(X)^{\prime}$ be the positive cone in $\mathcal{M} b(X)^{\prime}$, i.e., 
the dual cone of $S \in \mathcal{M} b(X)$. Convergence in the weak* topology of $\mathcal{H} b(X)^{\prime}$ is denoted by $\stackrel{w *}{\rightarrow}$.

If $X$ is a topological space, then $C(X)$ denotes the Banach space of all realvalued bounded continuous functions on $X$, and if $X$ is locally compact Hausdorff, $C_{0}(X)\left(C_{c}(X)\right.$ resp.) denotes the Banach space of real-valued continuous functions that vanish at infinity (with compact support, resp.).

In the sequel, the term measure will stand for a nonnegative $\sigma$-additive measure and a set function on $\mathscr{B}$ with the finite-additivity property (and not necessarily the $\sigma$ additivity property) will be referred to as a finitely additive measure. Let $M(X)$ be the Banach space of signed measures on $(X, \mathscr{B})$ equipped with the total variation norm $|\cdot|_{T V}$, simply denoted $|\cdot|$.

Note that $M(X) \subset \mathcal{M} b(X)^{\prime}$ and for every $f \in \mathcal{M} b(X), \mu \in M(X), \int f d \mu=\langle\mu, f\rangle$ when $\mu$ is considered to be an element of $\mathcal{M} b(X)^{\prime}$.

Also note that any element $\varphi \in S^{\prime}$ can be associated with a finitely additive nonnegative measure (also denoted $\varphi) \varphi(A):=\left\langle\varphi, 1_{A}\right\rangle, \forall A \in \mathfrak{B}$, so that $\varphi(A \cup B)=$ $\varphi(A)+\varphi(B), \forall A, B \in \mathscr{B}$ with $A \cap B=\emptyset$. Thus, $\varphi(A) \leq \varphi(X)=|\varphi|, \forall A \in \mathscr{B}$ (see e.g., $[3])$.

For a topological space $X$, by analogy with sequences of probability measures in a metric space, a sequence of measures $\left\{\mu_{n}\right\}$ in $M(X)$ is said to converge weakly to $\mu \in M(X)$, iff

$$
\int f d \mu_{n} \rightarrow \int f d \mu, \quad \forall f \in C(X)
$$

This type of convergence is denoted $\mu_{n} \stackrel{\text { weakly }}{\rightarrow} \mu$.

Similarly, and again, by analogy with sequences of probability measures in a metric space, if $X$ is a locally compact Hausdorff space, a sequence of measures $\left\{\mu_{n}\right\}$ in $M(X)$ is said to converge vaguely to $\mu \in M(X)$, iff

$$
\int f d \mu_{n} \rightarrow \int f d \mu, \quad \forall f \in C_{0}(X),
$$

and this type of convergence is denoted $\mu_{n} \stackrel{\text { vaguely }}{\rightarrow} \mu$. In fact, because the topological dual of $C_{0}(X)$ is $M(X)$ (see e.g., [1]), the vague convergence is simply the weak* convergence in $M(X)$.

A sequence $\left\{\mu_{n}\right\}$ in $M(X)$ is said to converge setwise to $\mu \in M(X)$ iff

$$
\mu_{n}(B) \rightarrow \mu(B), \quad \forall B \in \mathscr{B},
$$

and this convergence is denoted $\mu_{n} \stackrel{\text { setwise }}{\rightarrow} \mu$.

Finally, a sequence $\left\{\mu_{n}\right\}$ in $M(X)$ converges to $\mu \in M(X)$ in total variation (or convergences strongly (or in norm) to $\mu$ ) iff $\left|\mu_{n}-\mu\right| \rightarrow 0$ as $n \rightarrow \infty$. This convergence is denoted by $\mu_{n} \stackrel{T V}{\rightarrow} \mu$.

\section{Preliminaries}

In this section, we present some results that we will repeatedly use in the sequel.

For a nonnegative finitely additive measure $\mu$, proceeding as in [6], let:

$$
\Gamma(\mu):=\{\nu \in M(X) \mid 0 \leq \nu \leq \mu\}, \Delta(\mu):=\{\nu \in M(X) \mid \mu \leq \nu\},
$$


where by $\nu \leq \mu$ we mean $\nu(A) \leq \mu(A), \forall A \in \mathfrak{B}$.

Given two ( $\sigma$-additive) measures $\varphi$ and $\psi$, let

$\sup (\varphi, \psi)=\varphi \vee \psi:=\frac{|\varphi-\psi|+\varphi+\psi}{2} \inf (\varphi, \psi)=\varphi \wedge \psi:=\frac{\varphi+\psi-|\varphi-\psi|}{2}$

where for a signed measure $\gamma,|\gamma|$ is its corresponding total variation measure. With the partial ordering $\leq, M(X)$ is a complete Banach lattice (see e.g., [5]).

Lemma 3.1: Let $\mu$ be (nonnegative) finitely additive measure. Then,

(i) $\Gamma(\mu)$ has a maximal element $\varphi \in M(X)^{+}$.

(ii) If $\Delta(\mu) \neq \emptyset$, then $\mu$ is $\sigma$-additive.

Proof: To prove that $\Gamma(\mu)$ has a maximal element we use arguments similar to those in [6]. Let $\delta:=\sup _{\nu \in \Gamma(\mu)} \nu(X)$. Of course, we have $\delta \leq \mu(X)=1$. Thus, consider a sequence $\left\{\nu_{n}\right\}$ in $M(X)^{+}$, with $\nu_{n}(X) \uparrow \delta$. Define

$$
\varphi_{n}:=\nu_{1} \vee \nu_{2} \vee \ldots \vee \nu_{n}
$$

$\left\{\varphi_{n}\right\}$ is an increasing sequence in $\Gamma(\mu)$. Indeed, for any two measures $\tau$ and $\chi$ in $\Gamma(\mu),(\tau \vee \chi)(A) \leq \mu(A) \quad \forall A \in \mathfrak{B}$.

Since $\varphi_{n}(A) \leq \mu(A), \forall A \in \mathfrak{B}$, and $\varphi_{n}$ is increasing, it converges setwise to an element $\varphi \leq \mu$. That $\varphi$ is a ( $\sigma$-additive) measure follows from the fact that $\left\{\varphi_{n}\right\}$ is an increasing sequence (see e.g., [2]). It follows that $\varphi \in \Gamma(\mu)$ and $\varphi(X)=\delta$. We now prove that $\varphi$ is a maximal element of $\Gamma(\mu)$.

Consider any element $\chi \in \Gamma(\mu)$. Assume that there is some $A \in \mathfrak{B}$ such that $\chi(A)>\varphi(A)$. Let $\tau:=\chi \vee \varphi$. From the Hahn-Jordan decomposition of $(\chi-\varphi)$, $\exists X_{1}, X_{2}$ with $X_{1} \cup X_{2}=X, X_{1} \cap X_{2}=\emptyset$ so that

$$
\tau(A)=\chi\left(A \cap X_{1}\right)+\varphi\left(A \cap X_{2}\right), \quad A \in \mathfrak{B} .
$$

Thus, $\chi\left(X_{1}\right)>\varphi\left(X_{1}\right)$ and, therefore,

$$
\tau(X)=\chi\left(X_{1}\right)+\varphi\left(X_{2}\right)>\varphi\left(X_{1} \cup X_{2}\right)=\delta
$$

is a contradiction with $\tau \in \Gamma(\mu)$ and $\delta=\max \{\nu(X) \mid \nu \in \Gamma(\mu)\}$. Hence, $\chi \leq \varphi$. In fact, $\varphi$ is the $\sigma$-additive part in the decomposition of $\mu$ into a $\sigma$-additive part $\mu_{c}$ and a purely finitely additive part $\mu_{p}$, with $\mu=\mu_{c}+\mu_{p}$ (see [6]).

To prove $(i i)$, note that if $0 \leq \mu \leq \psi$, where $\psi$ is $\sigma$-additive, then $\mu$ is $\sigma$-additive (see e.g., [3], [6]). Indeed, for any decreasing sequence of sets $\left\{A_{n}\right\}$ in $\mathfrak{B}$ with $A_{n} \downarrow \emptyset$, we have $0 \leq \mu\left(A_{n}\right) \leq \psi\left(A_{n}\right) \downarrow 0$ which implies. $\mu\left(A_{n}\right) \downarrow 0$, i.e., $\mu$ is $\sigma$-additive.

Lemma 3.2: Let $\left\{\mu_{n}\right\}$ be a sequence of (nonnegative) $\sigma$-additive measures on $(X, \mathfrak{B})$ with $\sup _{n} \mu_{n}(X)<\infty$. Then,

$$
O-\liminf _{n \rightarrow \infty} \mu_{n}:=V_{k \geq 1}^{\vee} \hat{n}_{n} \mu_{n} \text { is a (finite) } \sigma \text {-additive measure. }
$$

If $\mu_{n} \leq \nu(\in M(X)) \forall n$, then,

$$
O-\limsup _{n \rightarrow \infty} \mu_{n}:={ }_{k \geq 1} \underset{n \geq k}{\vee} \mu_{n} \text { is a (finite) } \sigma \text {-additive measure. }
$$

Proof: Let $\varphi_{k n}:=\mu_{k} \wedge \mu_{k+1} \wedge \ldots \wedge \mu_{n} . \quad\left\{\varphi_{k n}\right\}_{n>0}$ is a decreasing sequence so that it converges setwise to a (finite) $\sigma$-additive measure $\varphi_{k}$ (see e.g., [4]). In turn, $\left\{\varphi_{k}\right\}$ is an increasing sequence with $\sup _{k} \varphi_{k}(X)<\infty$. Hence, $\varphi_{k}$ converges setwise to a (finite) $\sigma$-additive measure and (3.2) follows from 


$$
\stackrel{\vee}{\geq 1}_{n \geq k} \mu_{n}=\lim _{k \rightarrow \infty} \varphi_{k}
$$

(3.3) follows from $0 \leq \mu_{n} \leq \nu$ for all $n$ and the fact that $M(X)$ is a complete Banach lattice (see e.g., [5]).

In the sequel, to avoid confusion, the reader should be careful in distinguishing

$$
\left(O-\liminf _{n \rightarrow \infty} \mu_{n}\right)(B) \text { from } \liminf _{n \rightarrow \infty} \mu_{n}(B) \quad B \in \mathscr{B},
$$

for we have in fact,

$$
\left(O-\liminf _{n \rightarrow \infty} \mu_{n}\right)(B) \leq \liminf _{n \rightarrow \infty} \mu_{n}(B) \quad B \in \mathfrak{B}
$$

\section{Setwise Convergence}

We now give sufficient conditions for setwise convergence of an $F$-converging sequence, where $F$ is a subset of $\mathcal{M} b(X)$ separating points of $M(X)$ and such that $\mu \in M(X)$ and $0 \leq \int f d \mu \forall 0 \leq f \in F$ imply $\mu \geq 0$. Typical examples of such $F$ are $C(X)$ for a topological space $X$ and $C_{c}(X)$ or $C_{0}(X)$ for a locally compact Hausdorff space $X$.

Lemma 4.1: Let $F$ be a subspace of $\mathcal{M b}(X)$ separating points in $M(X)$ and such that for every $\mu \in M(X), 0 \leq \int f d \mu \quad \forall 0 \leq f \in F$ yields that $\mu \geq 0$. Let $\left\{\mu_{n}\right\}$ be a sequence of (nonnegative) measures on $(X, \mathfrak{B})$ with $\sup _{n} \mu_{n}(X)<\infty$. Assume that $\mu_{n} \stackrel{F}{\rightarrow} \mu \in M(X)$, i.e.,

$$
\int f d \mu_{n} \rightarrow \int f d \mu \quad \forall f \in F
$$

(i) If $\left(O-\liminf _{n} \mu_{n}\right)(X)=\mu(X)$ then $\mu_{n}^{\text {setwise }} \stackrel{\text {. }}{\rightarrow}$.

(ii) If for some $\nu \in M(X), \mu_{n} \leq \nu \forall n$, then $\mu_{n}^{\text {setwise }} \rightarrow$.

(iii) If $\left(O-\liminf _{n} \mu_{n}\right)(X)=\mu(X)=\left(O-\lim \sup _{n} \mu_{n}\right)(X)$ then $\mu_{n} \stackrel{T V}{\rightarrow} \mu$.

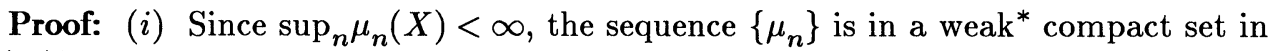
$\mathcal{M} b(X)^{\prime}$. Thus, there is a directed set $D$ and a subnet (not a subsequence in general) $\left\{\mu_{n}, \alpha \in D\right\}$ that converges to some $0 \leq \varphi$ in $\mathcal{M} b(X)^{\prime}$ for the weak ${ }^{*}$ topology in $\mathcal{M}_{b}\left({ }^{\alpha} X\right)^{\prime}$, and $\varphi$ is a finitely additive measure. From (3.2) in Lemma 3.2, $O-\liminf _{n} \mu_{n}$ exists and $\varphi \geq O-\liminf _{n} \mu_{n}$. Now, $\varphi$ has a unique decomposition into a $\sigma$-additive (nonnegative) part $\varphi_{c}$ and a purely finitely additive (nonnegative) part $\varphi_{p}$ with $\varphi=\varphi_{c}+\varphi_{p}$ (see e.g., [6]).

From $\sigma$-additivity of $O-\liminf _{n} \mu_{n}$ and $O-\liminf _{n} \mu_{n} \leq \varphi$, we have that $O-$ $\liminf _{n} \mu_{n} \leq \varphi_{c}$ since $\varphi_{c}$ is a maximal element of $\Gamma(\varphi)$ (see the proof of Lemma 3.1). Therefore, $\varphi_{c}(X) \geq\left(O-\liminf _{n} \mu_{n}\right)(X)=\mu(X)$. In addition,

$$
\langle\mu, f\rangle=\left\langle\varphi_{c}, f\right\rangle+\left\langle\varphi_{p} f\right\rangle \quad \forall f \in F .
$$

In particular, for $f \geq 0$ in $F$,

$$
\int f d\left(\mu-\varphi_{c}\right) \geq 0 \quad \forall 0 \leq f \in F
$$

Thus, $\mu \geq \varphi_{c}$ and $\mu(X)=\varphi_{c}(X)$ which in turn implies $\varphi_{c}=\mu$ and $\varphi_{p}(X)=0$, i.e., $\varphi$ is $\sigma$-additive and $\varphi=\mu$. As $\varphi$ was an arbitrary weak* accumulation point, all the weak $^{*}$ accumulation points are identical and equal to $\mu$, i.e., $\mu_{n} \rightarrow \mu$ for the weak* 
topology in $\mathcal{M b}(X)^{\prime}$ and, in particular, $\mu_{n}^{\text {setwise }} \rightarrow$.

(ii) As $\mu_{n} \leq \nu$ for all $n$, the sequence $\left\{\mu_{n}\right\}$ is in a weakly sequentially compact set of $M(X)$. Indeed, for all $n, \mu_{n}$ are norm-bounded and the $\sigma$-additivity of $\mu_{n}$ is uniform in $n$ (if $A_{k} \downarrow \emptyset, \nu\left(A_{k}\right) \rightarrow 0$ so that $\mu_{n}\left(A_{k}\right)\left(\leq \nu\left(A_{k}\right)\right) \rightarrow 0$ uniformly). Therefore, from Theorem 2, p. 306 in $[3],\left\{\mu_{n}\right\}$ forms a weakly sequentially compact set in $M(X)$. Hence, there is a subsequence $\left\{\mu_{n_{k}}\right\}$ that converges weakly to some $\varphi \in$ $M(X)$, and, in particular,

$$
\int f d \mu_{n_{k}} \rightarrow \int f d \varphi \quad \forall f \in \mathcal{M} b(X),
$$

so that, from the $F$-convergence of $\mu_{n}$ to $\mu$,

$$
\int f d \mu=\int f d \varphi \quad \forall f \in F
$$

As both $\varphi$ and $\mu$ are in $M(X)$ and $F$ separates points in $M(X),(4.3)$ implies $\mu=\varphi$. As $\varphi$ was an arbitrary weak-limit point of $\left\{\mu_{n}\right\}$ in $M(X)$, we also conclude that all the weak-limit points are all equal to $\mu$. In other words, $\mu_{n}^{\text {setwise }} \rightarrow$.

(iii) From (i) we conclude that $O-\liminf _{n} \mu_{n}=\mu$ and with similar arguments, $O-\lim \sup _{n} u_{n}=\mu$, i.e., the sequence $\left\{\mu_{n}\right\}$ in $M(X)$ has an $O$-limit $\mu$, or equivalently (see e.g., [5]), there exists $\left\{w_{n}\right\}$ in $M(X)$ such that

$$
\left|\mu_{n}-\mu\right| \leq w_{n} \text { with } w_{n} \downarrow 0
$$

Clearly, this implies convergence in total variation since

$$
\left|\mu_{n}-\mu\right|(X) \leq w_{n}(X) \text { with } w_{n}(X) \downarrow 0
$$

Lemma 4.1 applies to the following situations

- $\quad X$ is a locally Hausdorff space and $\mu_{n}^{\text {vaguely }} \rightarrow \in M(X)$, i.e.,

$$
\int f d \mu_{n} \rightarrow \int f d \mu \quad \forall f \in C_{0}(X)=: F
$$

or if

$$
\int f d \mu_{n} \rightarrow \int f d \mu \quad \forall f \in C_{c}(X)=: F
$$

- $\quad X$ is a topological space and $\mu_{n} \stackrel{\text { weakly }}{\rightarrow} \mu \in M(X)$, i.e.,

$$
\int f d \mu_{n} \rightarrow \int f d \mu \quad \forall f \in C(X)=: F .
$$

As a consequence of Lemma 4.1 we also get:

Corollary 4.2: Let $X$ be a locally compact Hausdorff space and $\lambda$ a $\sigma$-finite measure on $(X, \mathfrak{B})$. Consider a sequence of probability densities $\left\{f_{n}\right\} \in L_{1}(\lambda)$ with almost everywhere limit $f \in L_{1}(\lambda)$. Let

$$
\mu(B):=\int_{B} f d \lambda, \mu_{n}(B):=\int_{B} f_{n} d \lambda \quad B \in \mathscr{B}, \quad n=1, \ldots
$$

Assume that $\mu_{n}^{\text {vaguely }} \rightarrow$. If $\mu_{n} \leq \nu$ for some $\nu \in M(X)$ then: 


$$
\int\left|f_{n}-f\right| d \lambda \rightarrow 0 \text { as } n \rightarrow \infty
$$

and $\mu_{n} \rightarrow \boldsymbol{T V}$. In addition, if $\lambda$ is finite, the family $\left\{f_{n}\right\}$ is uniformly integrable.

Proof: Since the $\mu_{n}$ 's are order-bounded, from Lemma 4.1(ii) with $F:=C_{0}(X)$, we conclude that $\mu_{n}^{\text {setwise }} \rightarrow$. In particular, this implies that

$$
\int f_{n} d \lambda \rightarrow \int f d \lambda \text { as } n \rightarrow \infty
$$

and by Scheffe's Theorem,

$$
\int\left|f_{n}-f\right| d \lambda \rightarrow 0 \text { as } n \rightarrow \infty,
$$

which yields (4.8). That $\mu_{n} \stackrel{T V}{\rightarrow} \mu$ follows from the $L_{1}$ convergence of $f_{n}$ to $f$, i.e., (4.8). If $\lambda$ is finite, the uniform integrability of the family $\left\{f_{n}\right\}$ follows from [2], $\mathrm{p}$. 155.

Note that if instead of $\mu_{n} \leq \nu$, we had $\left|f_{n}\right| \leq g \in L_{1}(\lambda)$ then by the Dominated Convergence Theorem, $\int f_{n} d \lambda \rightarrow \int f d \lambda$ and (4.8) would follow from Scheffe's Theorem. However, note that the condition $\mu_{n} \leq \nu$ does not require $\nu$ to have a density.

\section{References}

[1] Ash, R.B., Real Analysis and Probability, Academic Press, New York 1972.

[2] Doob, J.L., Measure Theory, Springer-Verlag, New York 1994.

[3] Dunford, N. and Schwartz, J.T., Linear Operators Part I, J. Wiley, New York 1958.

[4] Getoor, R.K., Excessive Measures, Birkhäuser, Boston 1990.

[5] Yosida, K., Functional Analysis, Springer-Verlag, New York 1980.

[6] Yosida, K. and Hewitt, E., Finitely additive measures, Trans. Amer. Math. Soc. 72 (1952), 46-66. 


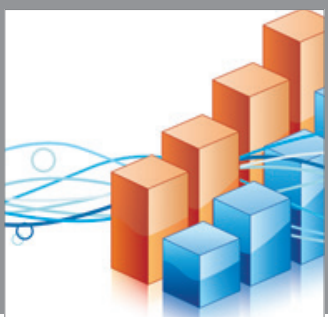

Advances in

Operations Research

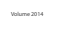

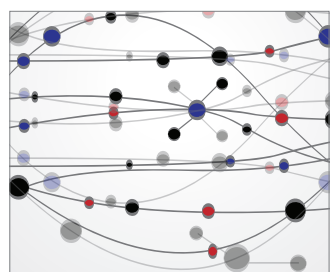

\section{The Scientific} World Journal
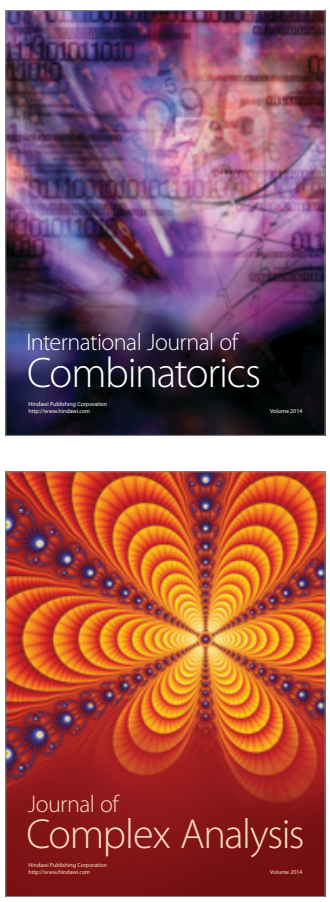

International Journal of

Mathematics and

Mathematical

Sciences
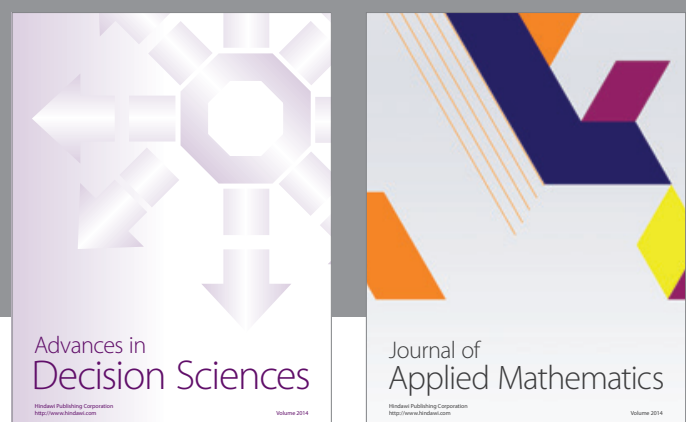

Journal of

Applied Mathematics
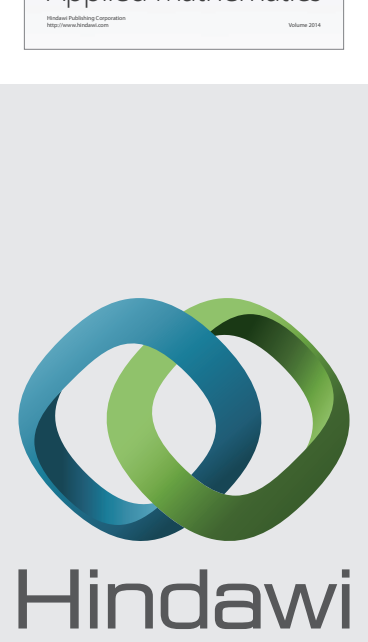

Submit your manuscripts at http://www.hindawi.com
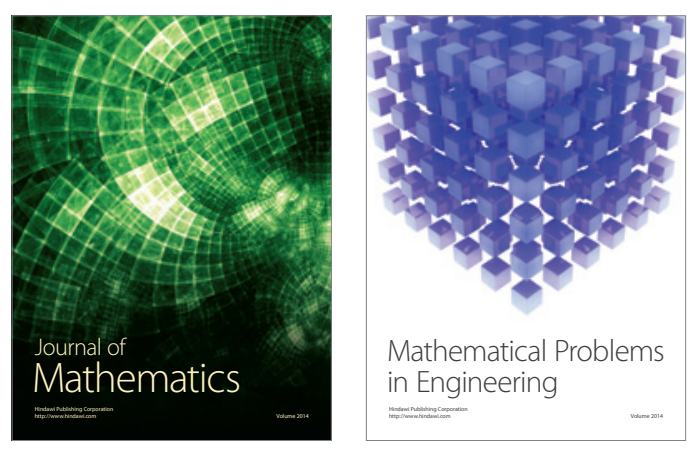

Mathematical Problems in Engineering
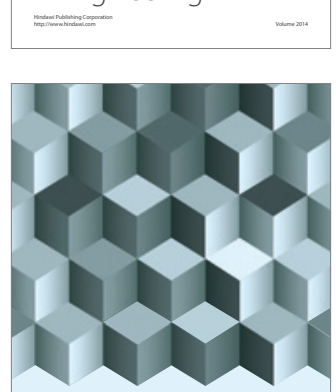

Journal of

Function Spaces
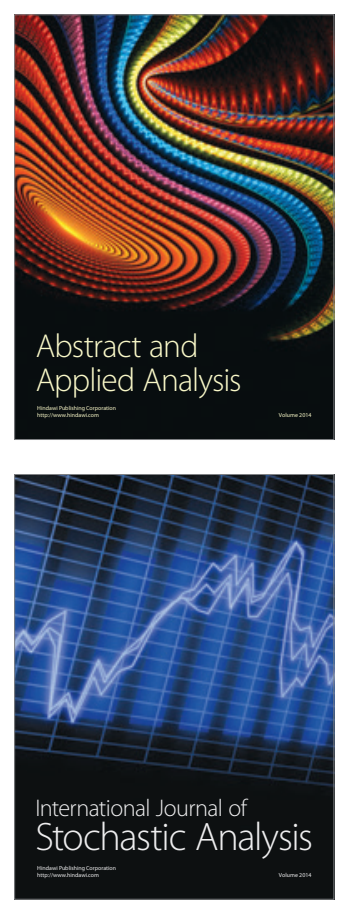

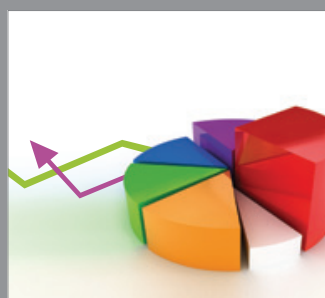

ournal of

Probability and Statistics

Promensencen
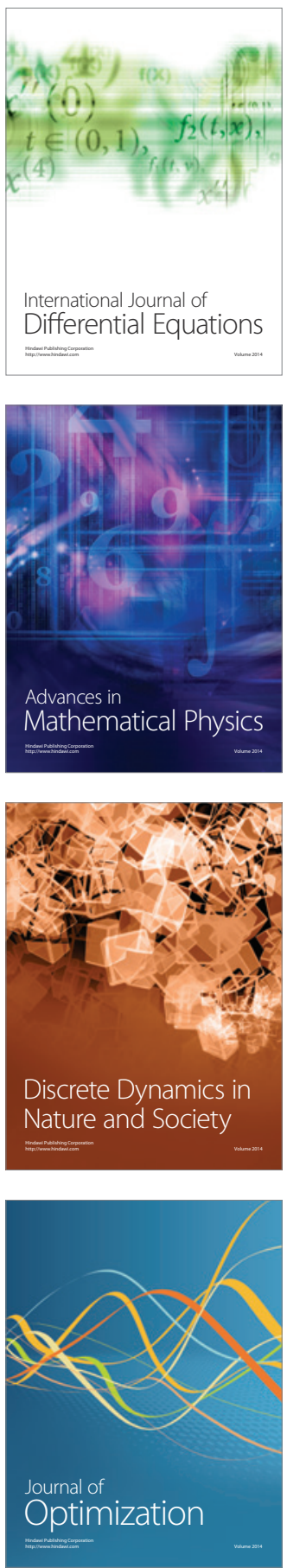